Proceedings of the International Symposium on Physics of Materials (ISPMA 14), September 10-15, 2017, Prague

\title{
Anisotropy of Thermal Expansion in an AZ31 Magnesium Alloy Subjected to the Accumulative Roll Bonding
}

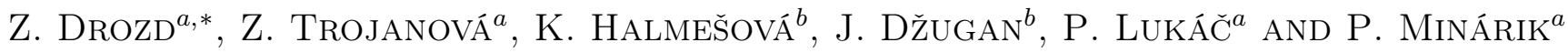 \\ ${ }^{a}$ Charles University Prague, Faculty of Mathematics and Physics, \\ V Holešovičkách 2, CZ-18200 Prague 8, Czech Republic \\ ${ }^{b}$ Comtes FHT, Dobřany, Průmyslová 996, 33441 Dobřany, Czech Republic
}

\begin{abstract}
Accumulative roll bonding is one of method for the production of metal materials with ultrafine grain microstructure. Sheets of the AZ31 magnesium alloy subjected to the 1 and 2 accumulative roll bonding passes through the rolling mill were cut so that the sample axis was either parallel or perpendicular to the rolling direction. The linear thermal expansion of samples was measured using a LINSEIS L75PT-1600 dilatometer in the temperature range from room temperature up to $400^{\circ} \mathrm{C}$. The thermal expansion curves of the sheets were measured during two consequent heating and cooling cycles. The temperature dependences of the relative elongation and of the thermal expansion coefficient were estimated from the dilatometer measurements. Planar anisotropy of the thermal expansion was found.
\end{abstract}

DOI: $10.12693 /$ APhysPolA.134.820

PACS/topics: 65.60.+a, 62.40.+i, 62.20.F-

\section{Introduction}

Accumulative roll bonding (ARB) is one of the severe plastic deformation (SPD) processes successfully used to obtain an ultrafine grain size or even nanocrystalline structure [1]. The principle of this method is repetitive rolling of a stack of metal sheets to a severe reduction ratio followed by cutting of the resulting sheet into two halves, putting them together and rolling them again. ARB technique has certain advantages in comparison with other SPD methods. It is cheaper than for instance ECAP method and relatively big sheets can be obtained using ARB. To the earliest publications dealing with ARB method belongs the paper published by Tsui et al. [2]. While mechanical properties of magnesium alloys submitted to ARB procedure were reported in several papers [3, 4], other physical properties were studied only rarely [5]. The aim of this study is to contribute to the knowledge and understanding of the linear thermal expansion of AZ31 sheets subjected to ARB procedure.

\section{Experimental}

Sheets of commercially available AZ31 magnesium alloy (3 wt\% Al, $1 \mathrm{wt} \% \mathrm{Zn}, 0.2 \mathrm{wt} \% \mathrm{Mn}$ - balance $\mathrm{Mg}$ ) with a thickness of $2 \mathrm{~mm}$ were subjected to the ARB process at $400^{\circ} \mathrm{C}$ with a rolling speed of $0.4 \mathrm{~m} / \mathrm{s}$. Material subjected to one such a pass through the rolling machine is hereafter labelled ARB 1, sheets subjected to two passes ARB 2, and as received sheets are designed

*corresponding author; e-mail: zdenek.drozd@mff.cuni.cz as ARB_0. Samples were cut so that the longer sample axis was either parallel (L-orientation) or perpendicular (T-orientation) to the rolling direction (RD).

The linear thermal expansion was measured using LINSEIS L75PT-1600 dilatometer with horizontal dual sample arrangement. Two temperature cycles (heatingcooling) were performed for each sample using heating and cooling rates of $1^{\circ} \mathrm{C} / \mathrm{min}$ in a resistance furnace in an argon protective atmosphere. Length changes were measured by linear variable differential transformer (LVDT) sensor through quartz push-rods in the temperature range from room temperature to $400{ }^{\circ} \mathrm{C} . \quad \mathrm{Al}_{2} \mathrm{O}_{3}$ reference was used for correction measurements. The microstructure samples was studied using a FEI Quanta 200 FX scanning electron microscope (SEM) equipped with EDAX EBSD system. The Young modulus was measured in the resonant frequency and damping analyser (RFDA). Samples were excited to vibrations in the resonant frequency using a small striker. Free vibrations of the sample in the bending mode were registered with a microphone and fast Fourier transform was used for estimation of the resonant frequency.

\section{Results and discussion}

Scanning electron micrographs of the sheets surface are shown in Fig. 1a-c. The rolled sheet (ARB 0) exhibits grain structure consisting of bigger grains which are surrounded by smaller grains as shown in Fig. 1a; Fig. 1b shows the grain refinement after the first pass through the rolling mill (ARB 1). Results of the second ARB process are shown in Fig. 1c. Almost uniform microstructure with fine grains is observed in this case.

Similar red colours of grains in Fig. 1 indicate that the material exhibit certain texture developed during rolling. 

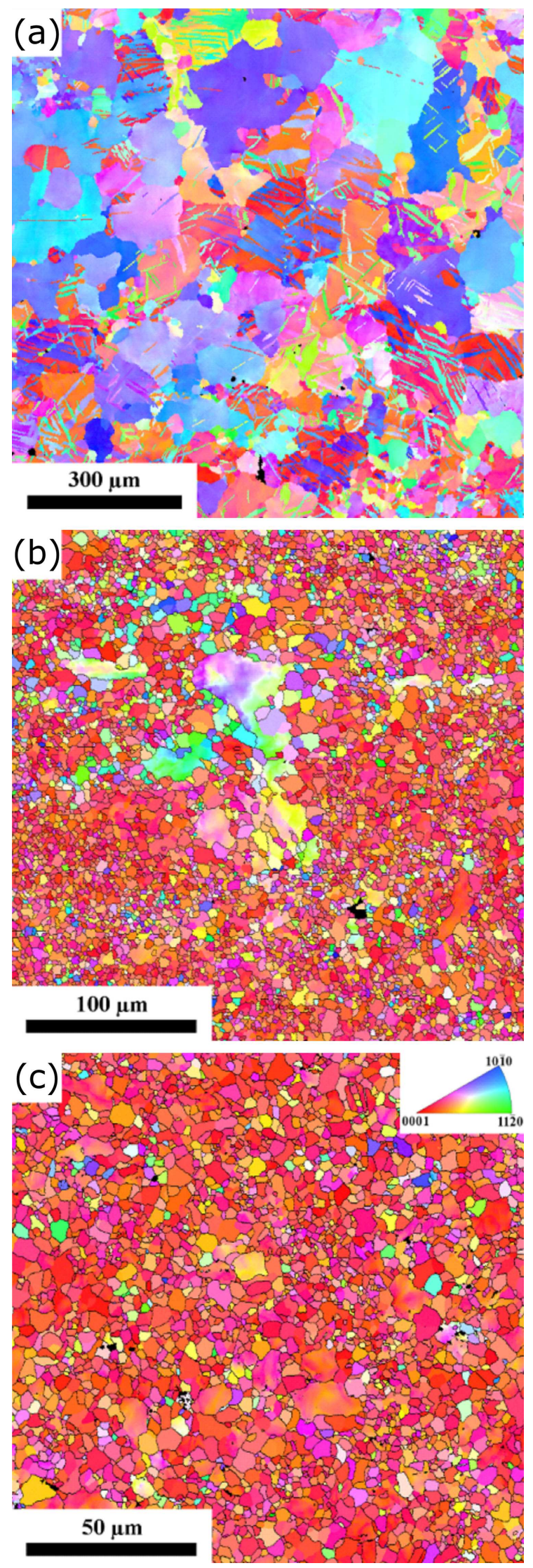

Fig. 1. Microstructure of the ARB_0 (a), ARB_1 (b), and ARB_2 samples (c).
Texture measurements of sheets showed that basal planes (0001) in grains are mostly oriented parallel to the sheet surface with the distribution of basal poles tilted away from the normal direction towards the transversal direction (perpendicular to the rolling direction in the sheet surface). Figures documenting this texture are reported in [5]. In this textured microstructure, the $\langle c\rangle$ axis of the hexagonal cell is perpendicular to the sheet surface. Permanent residual strain was observed after the first run in the dilatometer documented by plastic deformation occurring during the heating-cooling cycle. Permanent strain estimated in samples of the L orientation (expansion in the rolling direction) is higher than that estimated in samples with the $\mathrm{T}$ orientation (expansion perpendicular to the rolling direction) as shown in Fig. 2a and b. Permanent shortening of samples with the $\mathrm{T}$ orientation is practically the same for all samples. The Young modulus values measured at room temperature are shown in Table I. It can be seen that moduli are higher for the samples of $\mathrm{T}$ orientation and the values for both orientations decreased with increase of number of passes.

TABLE I

Young's modulus (YM) measured at room temperature and thermal expansion coefficient (CTE) extrapolated to room temperature.

\begin{tabular}{l|c|c|c}
\hline \hline Number of passes & 0 & 1 & 2 \\
\hline YM(L) [GPa] & 42.45 & 42.14 & 40.91 \\
YM(T) [GPa] & 45.30 & 43.84 & 42.14 \\
CTE(L) $\left[\times 10^{-6} \mathrm{~K}^{-1}\right]$ & $25.91_{5}$ & 26.05 & 25.04 \\
CTE(T) $\left[\times 10^{-6} \mathrm{~K}^{-1}\right]$ & 27.16 & 26.49 & 26.41
\end{tabular}

Planar plastic deformation during the hot rolling is realised by the movement of $\langle a\rangle$ dislocations with the Burgers vector of $1 / 3\langle 11 \overline{2} 0\rangle$ in basal planes in the rolling direction. Details of this mechanism were described indepth by Balík and co-workers in [6]. Moving dislocations in grains are stopped on obstacles and stored in pile-ups. With increase of number of passes the grain size decreases then the mean free path of dislocations is also lower and tendency to form pile ups increases. Owing to special grains orientations done by the texture, dislocation pile-ups are formed previously in the rolling direction. Stored dislocations represent significant internal stresses. During the CTE measurements, the samples are subjected to thermal cycles. While heating back stress from pile ups presses stored dislocations back, dislocations may be partially annihilated. This mechanism leads to the sample shortening. This explanation may be supported by results of Stráská and co-workers [7] who studied thermal stability of AZ31 alloy subjected to EXECAP procedure using positron lifetime spectra. Specimens in their investigation were subjected to isochronal annealing at various temperatures. They found out that the dislocation density decreases with increasing annealing temperature. We suppose that similar decrease in the dislocation density occurred probably also in our samples during the thermal cycles. 


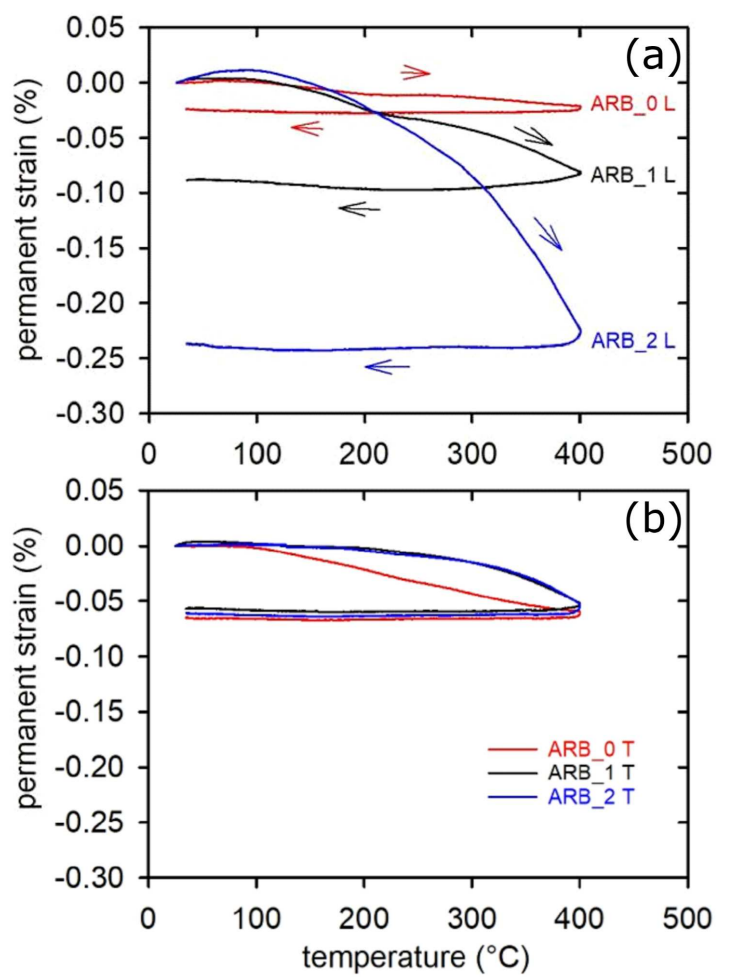

Fig. 2. Permanent strain measured for L samples (a) and $\mathrm{T}$ samples (b).
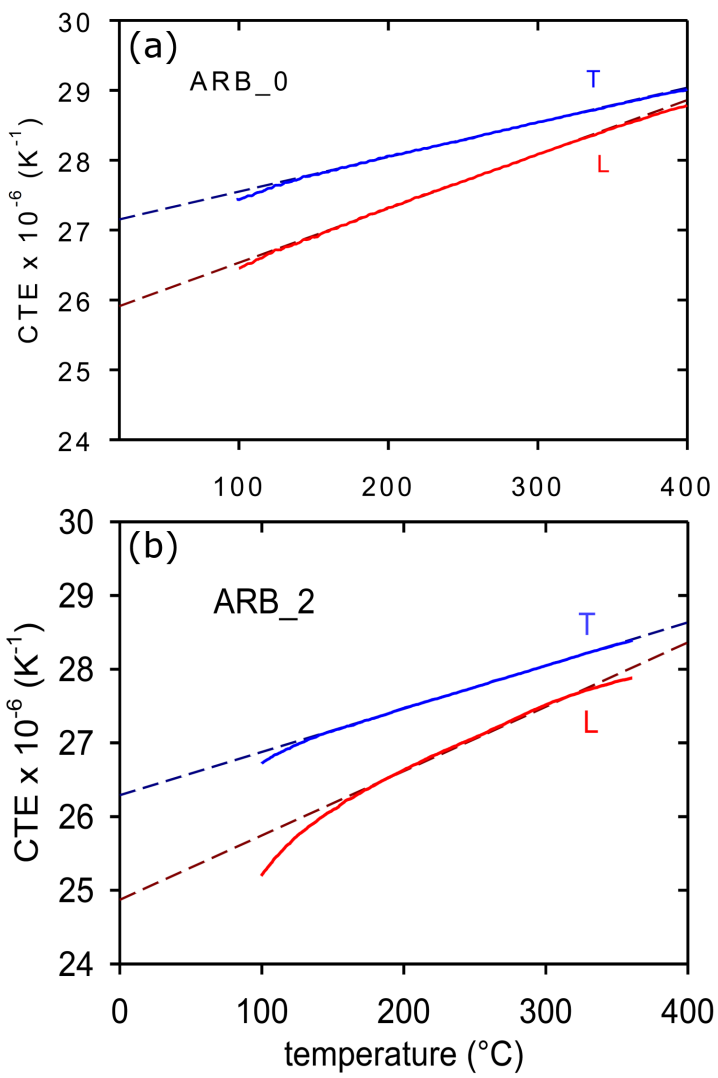

Fig. 3. Temperature dependences of the thermal expansion coefficient estimated for as-received sheet (a) and sheet after two rolling passes (b).
The temperature dependencies of CTE for specimens ARB_0 and ARB_2 are presented in Fig. 3a and b. The $\bar{C}$ TE increases with increasing temperature. Lower CTE values for L-orientation, and different slopes of the dependences obtained for $\mathrm{L}$ and $\mathrm{T}$ oriented samples, are common features of both (ARB_0 and ARB_2) samples.

Similar results were estimated for ARB 1 samples. Also in this case, the CTE of L sample is lower than the CTE of T sample. The ARB process decreases the CTE values, see in Table I, where the CTE values extrapolated to temperature of $20^{\circ} \mathrm{C}$ are tabulated. The thermal expansion of single crystals estimated by the X-ray measurements of lattice constants showed that expansion in the $a$-axis direction is lower than that in the $c$-axis of the hexagonal cell [8]:

$$
\begin{aligned}
& \langle a\rangle=0.32075+[7.045(T-20) \\
& \left.+0.0047(T-20)^{2}\right] \times 10^{-6} \mathrm{~nm}, \\
& \langle c\rangle=0.5276+[11.758(T-20) \\
& \left.\quad+0.0080(T-20)^{2}\right] \times 10^{-6} \mathrm{~nm},
\end{aligned}
$$

where $T$ is temperature in ${ }^{\circ} \mathrm{C}$. Repeated rolling in the first and second pass improved the texture i.e. with increase of number of passes basal planes in more grains are parallel to the sheet surface with the $\langle a\rangle$ axes in the rolling direction. The elongation of lattice distance between the parallel $\{11 \overline{2} 0\}$ second order prismatic planes in the L sample is estimated. In the case of $\mathrm{T}$ samples, changes of distances between parallel first order $\{10 \overline{1} 0\}$ prismatic planes are considered. Planar anisotropy of the thermal expansion coefficient indicates possible anisotropy of various directions in the basal plane.

It should be noted that in the quasi-harmonic Debye model, the thermal expansivity, $\alpha$, is described as

$$
\alpha=\frac{\gamma C_{p}}{K_{s} V}=\frac{\gamma C_{v}}{K_{T} V},
$$

where $\gamma$ is the Grüneisen parameter, $V$ - the volume, $K_{T}$ - the isothermal bulk modulus, $K_{S}$ - the adiabatic bulk modulus and $C_{V}, C_{P}$ are the heat capacities at constant volume and pressure, respectively [9]. In the high temperature approximation (for $T \gg \Theta_{D}$, where $\Theta_{D}$ is the Debye temperature and the Poisson ratio is independent of volume), the Grüneisen parameter may be expressed by the following relation [10]:

$$
\gamma=\frac{1}{2} \frac{\mathrm{d} K}{\mathrm{~d} P}-\frac{1}{2}
$$

where $P$ is the pressure.

The ARB procedure decreased the grain size. Intensive plastic deformation introduced into the sheets also many new crystal defects, grain boundaries and interfaces which increased the volume, weakened the atomic bonding and changed the vibrational frequencies. 


\section{Conclusions}

The linear thermal expansion of AZ31 sheets subjected to the accumulative roll bonding was experimentally studied. From these results following points may be concluded:

- The ARB process refined the grain structure of AZ31 sheets.

- Permanent shortening of samples was observed after the first thermal cycle. Permanent residual strain depends on the number of the ARB passes in the sample with the orientation parallel to the rolling direction while it is constant in the transversal direction

- The CTE of the AZ31 sheets exhibit a planar anisotropy after rolling.

- The CTE decreases with increase of number of ARB passes.

\section{Acknowledgments}

This study was realised with the support of the Czech Science Foundation of the Czech Republic under the contract 107-15/11879S.

\section{References}

[1] Y. Saito, H. Utsunomiya, N. Tsuji, T. Sakai, Acta Mater. 47, 579 (1999).

[2] N. Tsuji, Y. Saito, S.H. Lee, Y. Minamino, Adv. Eng. Mater. 5, 338 (2003).

[3] W.J. Kim, J.B. Lee, W.Y. Kim, H.T. Jeong, H.G. Jeong, Scr. Mater. 56, 309 (2007).

[4] M.-Y. Zhan, Y.-Y. Li, W.-P. Chen, Trans. Nonferr. Met. Soc. China 18, 309 (2008).

[5] Z. Trojanová, J. Džugan, K. Halmešová, G. Németh, P. Minárik, P. Lukáč, Acta Phys. Pol. A 134, 863 (2018).

[6] P. Lukáč, J. Balík, Z. Trojanová, Kovove Mater.Metall. Mater. 49, 385 (2011).

[7] J. Stráská, M. Janeček, J. Čížek, J. Stráský, B. Hadzima, Mater. Character. 94, 69 (2015).

[8] P. Lukáč, in: Magnesium Technology: Metallurgy, Design Data, Applications, Eds. H. Friedrich, B.L. Mordike, Springer, Berlin 2006, p. 63.

[9] E. Grüneisen, Ann. Phys. 39, 257 (1912).

[10] A.Vijay, Indian J. Pure Appl. Phys. 49, 825 (2011). 